\title{
ANÁLISE E REDESENHO DOIS PROCESSOS NA CADEIA LOGÍSTICA DE SUPRIMENTO DE CALCÁRIO COMO INSUMO
}

\author{
R. L. BALDAM ${ }^{1}$, A. F. LOURENÇÃO ${ }^{2}$, D. M. SIMMER ${ }^{3}$, L. C. $\operatorname{COSTA}^{4}$, E. C. G. DA ROS ${ }^{5}$, T. DE P. COELHO JUNIOR \\ Universidade Federal do Espírito Santo \\ diogosimmer@gmail.com ${ }^{3}$
}

Submetido 21/12/2018 - Aceito 26/03/2020

DOI: $10.15628 /$ holos.2020.8098

\section{RESUMO}

O objetivo deste artigo é, através de indicadores, verificar os ganhos obtidos com a implementação de modelagem de processos na cadeia logística que transporta o calcário para sua utilização como insumo na siderurgia. Assim, buscou-se identificar os desperdícios existentes nos processos, através de uma investigação empírica do estudo de caso que observou grande perda de eficiência no fluxo produtivo que utiliza o calcário para produção de pelotas de minério de ferro. A implementação da modelagem foi realizada utilizando a notação BPMN (Business Process Modeling and Notation), quantificando os ganhos e relatando os problemas encontrados. Ficou evidenciado, nos resultados do presente estudo, uma redução de $80 \%$ nas paradas dos moinhos, por falta de insumo, resultando na melhora da operação logística de suprimento de calcário como insumo na pelotização do minério de ferro.

PALAVRAS-CHAVE: Business Process Management (BPM), Business Process Modeling and Notation (BPMN),

Gestão de Operações e Logística, calcário.

\section{ANALYSIS AND REDESIGN OF TWO PROCESSES IN THE LIMESTONE SUPPLY LOGISTICS CHAIN AS INPUT}

\begin{abstract}
The objective of this article is through indicators, to verify the gains obtained with the implementation of process modeling in the logistics chain that transports limestone for use as an input in the steel industry. Thus, we sought to identify the existing waste in the processes, through an empirical investigation of the case study that observed a great loss of efficiency in the production flow that uses limestone for the production of iron ore pellets. The
\end{abstract}

modeling implementation was performed using the BPMN (Business Process Modeling and Notation) notation, quantifying the gains and reporting the problems encountered. The results of the present study showed an $80 \%$ reduction in mill stops due to lack of inputs. Resulting in the improvement of the logistic operation of limestone supply as an input in the iron ore pelletizing. 


\section{INTRODUÇÃO}

O calcário é uma matéria prima utilizada em diversos setores produtivos, como na produção de cimento e cal, correção do solo na agricultura, fundente em metalurgia, fabricação de vidro e rochas ornamentais. A sua aplicação pode ser feita inclusive na diminuição de resíduos sólidos e elaboração de tijolos (Faé Gomes, Vilela, Zen, \& Osório, 2013).

Em uma das aplicações do setor siderúrgico, o calcário é misturado ao minério de ferro e outros aditivos para a produção de pelotas, que são utilizadas na produção de ferro gusa. No processo produtivo objeto desse estudo, a variabilidade do consumo de calcário é alta, a previsibilidade é baixa e o estoque interno é pequeno, o que ocasiona grandes gargalos na cadeia de seu abastecimento. $O$ transporte rodoviário é mobilizado e desmobilizado de acordo com a demanda no volume do insumo consumido. A identificação dos padrões da variabilidade, a simplificação das tarefas através da modelagem e a produção de modelos de processo estruturados auxiliam a restringir o fluxo de trabalho e definir a ação a ser tomada em cada situação (Yousfi, Saidi, \& Dey, 2016).

Delimita-se o processo logístico do insumo como sendo a programação de entrega da matéria-prima junto aos fornecedores e o recebimento e descarga dos caminhões. Frequentemente, são observados erros de comunicação que impactam no descarregamento dos caminhões com os insumos.

Gastos excessivos com logística e atraso na entrega são problemas frequentemente enfrentados pelas empresas. Estes problemas, além de impactarem diretamente na operação, têm os seus reflexos no setor financeiro, onerando o custo operacional (Souza et al., 2017).

O objetivo deste artigo é utilizar os conceitos e métodos do BPM (Business Process Management) na identificação das perdas no processo e assim estruturar e modelar o fluxo produtivo a ser seguido no transporte logístico do calcário como insumo. Neste contexto, surge a questão problema deste artigo: quais seriam os ganhos obtidos na modelagem do processo logístico do calcário como agente fundente para a pelotização de minério de ferro?

\section{REVISÃO BIBLIOGRÁFICA}

\subsection{Métodos de modelagem em processos}

O Gerenciamento de Processos de Negócios é uma técnica de gestão contemporânea que busca gerenciar as operações de uma organização em termos de processos de negócios, ou seja, conjuntos estruturados e controlados de atividades projetadas para produzir um resultado específico, destinado a um cliente ou mercado particular. O BPM fornece os métodos, ferramentas e técnicas para identificar, analisar, executar, monitorar e modificar o portfólio de processos de negócios da organização, resultando em um ciclo de melhoria contínua (Dijkman, Lammers, \& de Jong, 2016).

Para (Conforti, De Leoni, La Rosa, Van Der Aalst, \& Ter Hofstede, 2015), a utilização da ferramenta BPM auxilia na identificação dos riscos dos processos, apoia a tomada decisão, 
minimizando assim os possíveis riscos e a sua gravidade no processo, mapeando e mitigando possíveis falhas.

(Ko, Lee, \& Lee, 2009) enfatizam que o BPM se torna especialmente importante na atualidade devido a inúmeros fatores, tais como: aumento de fluxo de mercadorias, necessidade de rápida transferência de informação, rápida tomada de decisão, necessidade para adaptação das mudanças de demanda e maior número de competidores internacionais.

O BPM se confirmou como disciplina madura na última década, quando se comprovou sua eficácia, através dos conjuntos claramente definidos de princípios, métodos e ferramentas que compatibilizam conhecimento de diferentes áreas como, tecnologia da informação, ciências de gestão e engenharia industrial com o objetivo de melhorar vários processos, não somente os de negócio (Van Der Aalst, La Rosa, \& Santoro, 2016). A importância da modelagem de processos de negócio, etapa fundamental do BPM, tem sido mencionada desde os anos de 1960, contudo, apenas no início da década de 1990 passou-se a dar importância aos processos nas organizações (Aguilar-Savén, 2004).

Impulsionados pelo movimento de Qualidade Total, foi reforçada a partir do momento em que as empresas iniciaram o que ficou conhecido como BPR (Business Process Reengineering). No entanto, esta iniciativa de modelagem de processos não atendeu ao que se propusera. (Hammer, 1993) sugere que menos de $70 \%$ dos projetos de reengenharia obtiveram sucesso. Vários autores discursaram sobre o tema. (Launonen \& Kess, 2002) afirmaram que a razão para a falha dos esforços de reengenharia foi a falta de penetração nos níveis mais profundos da organização. (Yen, 2009) atribuiu o insucesso do BPR à falta de mensuração da eficácia dos processos.

(Ko et al., 2009; Vergidis, Turner, \& Tiwari, 2008) ressaltam que o BPM difere do BPR, pois não objetiva mudanças revolucionárias como este, mas sim a evolução contínua dos processos.

(Borges, Walter \& Santos 2016) afirmam que a revolução da tecnologia da informação deu força à utilização do BPM, abrindo a possibilidade de integração dos sistemas de informação entre as empresas, exigindo assim um trabalho de análise e redesenho para o funcionamento eficiente dos sistemas integrados.

(Vom Brocke et al., 2014) destacam que conceitos relacionados ao BPM, como o BPR, podem fornecer informações valiosas transferíveis para o conceito de BPM.

Ao longo desse desenvolvimento, também foram construídos diferentes métodos de modelagem de processos. (Aguilar-Savén, 2004) listou os principais métodos: fluxograma, diagrama de fluxo de dados, diagrama de papel de atividade (RAD), diagrama de papel de interação (RID), definição integrada para função de modelagem (IDEF), Petri-net colorida (CPN), método orientado ao objeto (OO).

(Rohleder \& Silver, 1997; Rosemann, 2006) enfatizaram a economia proporcionada pelo BPM (Business Process Management) e, segundo (Luo \& Tung, 1999), a modelagem de processos facilita o entendimento do processo por um grupo, utilizando uma representação padrão e é base para a melhoria e gerenciamento de processos.

Segundo Vom Brocke, existem dez princípios que devem ser seguidos e orientam o uso bem-sucedido do BPM na organização. Quando se implementa o BPM, os princípios são um conjunto de capacidades que auxiliam na compreensão e solução de desafios contemporâneos e 
futuros, conforme exposto na Tabela 1.

Tabela 1: Dez princípios para implantação do BPM.

\begin{tabular}{|c|c|c|}
\hline No. & Princípios & Descrição da manifestação positiva (+) e negativa (-) \\
\hline 1 & $\begin{array}{l}\text { Princípio da Conscientização do } \\
\text { Contexto }\end{array}$ & $\begin{array}{l}\text { + BPM deve se adequar ao contexto organizacional. } \\
\text { - Não deve seguir uma abordagem de livro de receitas. }\end{array}$ \\
\hline 2 & Princípio da Continuidade & $\begin{array}{l}\text { + BPM deve ser uma prática constante. } \\
\text { - Não deve ser um projeto único. }\end{array}$ \\
\hline 3 & Princípio da Ativação & $\begin{array}{l}\text { + BPM deve desenvolver capacidades. } \\
\text { - Não deve ser limitado ao combate a incêndios. }\end{array}$ \\
\hline 4 & Princípio do Holismo & $\begin{array}{l}\text { + BPM deve ser inclusivo no escopo } \\
\text { - Não deve ter um foco isolado. }\end{array}$ \\
\hline 5 & Princípio da Institucionalização & $\begin{array}{l}\text { + BPM deve ser incorporado na estrutura organizacional. } \\
\text { - Não deve ser uma responsabilidade ad-hoc. }\end{array}$ \\
\hline 6 & Princípio do Envolvimento & $\begin{array}{l}\text { + BPM deve integrar todos os grupos de stakeholders. } \\
\text { - Não deve negligenciar a participação dos funcionários. }\end{array}$ \\
\hline 7 & $\begin{array}{l}\text { Princípio da Compreensão } \\
\text { Conjunta }\end{array}$ & $\begin{array}{l}\text { + BPM deve criar um significado compartilhado. } \\
\text { - Não deveria ser a linguagem dos especialistas. }\end{array}$ \\
\hline 8 & Princípio do Propósito & $\begin{array}{l}\text { + BPM deve contribuir para a criação de valor estratégico. } \\
\text { - Não deve ser feito por uma questão de fazê-lo. }\end{array}$ \\
\hline 9 & Princípio da Simplicidade & $\begin{array}{l}\text { + BPM deve ser econômico. } \\
\text { - Não deve ser superengenharia. }\end{array}$ \\
\hline 10 & $\begin{array}{l}\text { Princípio da Apropriação } \\
\text { Tecnológica }\end{array}$ & $\begin{array}{c}\text { + BPM deve fazer uso oportuno da tecnologia. } \\
\text { - Não deve considerar o gerenciamento de tecnologia como um } \\
\text { pensamento posterior. }\end{array}$ \\
\hline
\end{tabular}

Fonte: Elaboração própria, com base em (Vom Brocke et al., 2014)

A modelagem dos processos permite abstrair e assim identificar como o trabalho é estruturado de forma simples em organizações complexas, apresentando modelos de fácil interpretação e auxiliando no entendimento dos sistemas e do papel de cada funcionário na organização(Corradini et al., 2018).

BPMN (Business Process Modeling and Notation) é uma notação padrão relativamente recente para BPM. É um padrão gráfico, desenvolvido por um consórcio de empresas, que permite aos usuários expressar o fluxo da informação, os pontos de decisão e os papéis funcionais dos processos de negócio através de um diagrama. Notadamente, os padrões gráficos são os mais fáceis de serem lidos e compreendidos mesmo sem treinamento técnico(Ko et al., 2009). 
(Recker, 2010; Vergidis et al., 2008) afirmam que o BPM necessita de padrões, tais como o BPMN, que proporcionem construções que sejam simples e de fácil comunicação. (Ko et al., 2009) complementou essa afirmação dizendo que os elementos do BPMN são parecidos com os do fluxograma, mas oferecem um controle de fluxo semântico muito mais preciso.

O BPMN é uma linguagem padrão que representa em uma forma gráfica os processos que ocorrem nas organizações permitindo que indivíduos com funções diferenciadas sejam capazes de interpretar de forma clara e fácil o processo (Corradini et al., 2018).

Devido às suas características, pode ser utilizado em diversos tipos de aplicações, desde ferramentas de modelagem simples até gerenciamento de processos de negócios com funcionalidades e características complexas (Solís-Martínez, Espada, Pelayo G-Bustelo, \& Lovelle, 2014).

Por exemplo, (Conforti, Dumas, García-Bañuelos, \& La Rosa, 2016; Mitsyuk, Shugurov, Kalenkova, \& van der Aalst, 2017) citam que, recentemente, várias técnicas de mineração de processos baseadas em BPMN foram propostas. Essas técnicas permitem representar processos, descobertos a partir dos logs de eventos de sistemas de informação sensíveis ao processo, de forma conveniente, utilizando o padrão BPMN. Para testar essas abordagens de mineração é necessária uma ferramenta apropriada para a geração de logs de eventos a partir de modelos BPMN.

Em contrapartida, a modelagem de processos ainda é criticada por consumir muito tempo para ser realizada, ser custosa (há investimentos em ferramentas, tecnologias, treinamentos) e não agregar valor suficiente (Rosemann, 2006; Vergidis et al., 2008). Segundo (Aguilar-Savén, 2004; Luo \& Tung, 1999), para selecionar o método de modelagem a ser empregado deve-se avaliar os objetivos da modelagem e suas perspectivas.

\subsection{Formatação da equipe de modelagem}

Diversos autores discursaram sobre a importância desta etapa. (Rohleder \& Silver, 1997) afirmam que a utilização de equipe, e não indivíduos, nas melhorias de processos é imperativo por dois motivos: processos envolvem muitas pessoas e a criatividade é alavancada em equipe; e complementou afirmando que os membros da equipe devem estar envolvidos no processo a ser modelado. (Launonen \& Kess, 2002) relataram quais são as competências necessárias na equipe para se obter sucesso na modelagem e complementaram que problemas irão ocorrer caso os membros da equipe não tenham o conceito claro das suas funções.

(Van Der Aalst et al., 2016) afirmam que ao se realizar a modelagem de um processo de negócio, a sua análise pode ser feita pela equipe simulando alternativas e assim fomentando ideias sobre como melhorar o processo, reduzindo os custos e aumentando os níveis de serviços.

\subsection{Indicadores de processo}

Os benefícios advindos da modelagem de processos só podem ser explicitados através do estabelecimento de parâmetros para verificação da situação atual e futura. (Rohleder \& Silver, 1997; Yen, 2009) concordam que a definição de indicadores de processo deve ser encarada como prioridade, pois eles são a base para o gerenciamento do processo. (Rosemann, 2006) aponta que 
é importante que se tenha, desde o início, o entendimento dos parâmetros que se deseja monitorar. Estes parâmetros devem ser mensuráveis e o processo de mensuração deve estar bem entendido desde o princípio. Deste modo, criam-se baselines através das quais as mudanças podem ser visualizadas.

Segundo (Rohleder \& Silver, 1997), para estabelecer os indicadores, primeiramente precisase identificar os objetivos do processo. As variáveis serão estabelecidas após acordo entre todos os participantes. Vale lembrar, contudo, que na maioria dos casos, o tempo é a variável de mensuração do desempenho do processo (Vergidis et al., 2008).

A multidimensionalidade na construção do BPM, quando modelamos processos apontam que os instrumentos de medida e indicadores devem ser relevantes e claros para que se tenha então o aumento da eficácia e efetividade nos processos organizacionais, levando assim ao aprimoramento e inovação (Schmiedel, Müller, \& vom Brocke, 2018).

(Vergidis et al., 2008) também salientam que todo processo deve ter um dono, senão a sua gestão se torna fragmentada e seus resultados ficam nebulosos.

É importante ressaltar que, devido à natureza qualitativa da modelagem de processos, análises quantitativas e avaliações são difíceis de serem implementadas (Vergidis et al., 2008). Deste modo, a utilização de questionários é vantajosa para quantificação de parâmetros subjetivos, como a satisfação dos usuários (Yen, 2009).

Finalmente, (Rohleder \& Silver, 1997) lembram que o ato de coletar dados é uma atividade que não agrega valor e só deve ser realizada se conduz a uma futura melhoria de processo.

\subsection{As-Is versus To-be}

(Rosemann, 2006) afirma que o resultado da modelagem não é apenas o modelo, mas também o processo de modelagem. $O$ ato de modelar é um gatilho para uma reação em cadeia que incrementa o entendimento do processo.

Um cuidado que o modelador deve ter é de se concentrar em capturar o modelo As-Is, ao invés do As-If (Rosemann, 2006). Ao término do modelo As-Is, ter-se-á o conhecimento necessário para modelar o processo otimizado To-Be. Para (Rohleder \& Silver, 1997), o primeiro passo para construção do To-Be é chamado de streamlining, que consiste na eliminação de atividades que não agregam valor.

(Vom Brocke et al., 2014) ressaltam que, no princípio da Compreensão conjunta, modelos de processo devem ser simples e intuitivos, dessa forma as organizações podem criar um significado compartilhado e um entendimento comum entre todas as partes envolvidas no processo. (Rosemann, 2006) compartilha desta ideia quando diz que, se um modelo não cabe em uma página, não é um bom modelo. Achar o nível ideal de detalhamento é um dos maiores desafios da modelagem. $O$ autor ainda listou os principais problemas encontrados na modelagem de processo: (i) processos de modelagem não conectados com pontos estratégicos da organização; (ii) falta de governança; (iii) falta de sinergia entre os diferentes departamentos da organização; (iv) falta de qualificação dos modeladores; (v) falta de representantes do processo; (vi) excesso de detalhes; (vii) falta de criatividade; e (viii) modelagem voltada para TI. 
Ressalta-se ainda que, de acordo com (Rohleder \& Silver, 1997), após a mudança do processo, é certo que haverá um período transiente no qual o processo pode piorar. Este período é parte do processo de aprendizagem.

\section{METODOLOGIA}

A estratégia escolhida para a pesquisa foi o Estudo de Caso, que, segundo Yin (1989), tem por objetivo examinar a fundo um fenômeno contemporâneo, em que o investigador tem pouco controle sobre os eventos. Neste caso, conduziu-se, empiricamente, uma pesquisa de fenômenos no seu contexto real e os resultados obtidos poderão servir de base de dados para estudos envolvendo o Gerenciamento de Processos de Negócios.

A pesquisa realizada tem natureza descritiva, já que o escopo do estudo se limita a descrever e informar sobre situações, fatos e dados coletados. Os dados coletados são quantitativos e foram utilizados para mensuração dos indicadores de processo propostos. Para a análise dos dados, limitou-se à utilização de ferramentas estatísticas básicas, como gráfico em barras e média aritmética.

Este artigo seguiu a seguinte trajetória: (i) estabelecimento dos indicadores do processo e da forma de mensuração; (ii) medição dos indicadores do processo atual; (iii) modelagem do processo atual (As-Is) e do processo otimizado (To-Be); (iv) implementação do novo processo; (v) medição dos indicadores do novo processo; e (vi) comparação com os dados coletados no processo As-Is. Na Tabela 2, pode ser observada a matriz de designe de Choguill que relaciona os objetivos e as ferramentas utilizadas para o seu atingimento.

Tabela 2: Apresentação metodológica dos objetivos, instrumentos e ferramentas utilizadas.
Objetivo Geral
Objetivos Específicos
Instrumentos e Ferramentas

\begin{tabular}{|c|c|c|}
\hline \multirow{5}{*}{$\begin{array}{c}\text { Medir ganhos } \\
\text { obtidos com a } \\
\text { modelagem do } \\
\text { processo da } \\
\text { logística de } \\
\text { calcário. }\end{array}$} & $\begin{array}{c}\text { Estabelecer e medir os } \\
\text { indicadores do processo. }\end{array}$ & $\begin{array}{l}\text { - Grupo de foco (setorial) - } \\
\text { Evento KAIZEN. }\end{array}$ \\
\hline & $\begin{array}{l}\text { - Descrever o processo atual de } \\
\text { planejamento e recebimento de } \\
\text { calcário }(A s-I s) \text {, }\end{array}$ & $\begin{array}{l}\text { • Business Process Model and } \\
\text { Notation - BPMN (OMG, 2013) } \\
\text { - Software MS-Visio }\end{array}$ \\
\hline & $\begin{array}{c}\text { - Descrever o processo otimizado } \\
\text { de planejamento e recebimento } \\
\text { de calcário }(T o-B e) \text {. }\end{array}$ & $\begin{array}{l}\text { - Business Process Model and } \\
\text { Notation - BPMN (OMG, 2013) } \\
\text { - Software MS-Visio }\end{array}$ \\
\hline & $\begin{array}{l}\text { - Implementar o processo } \\
\text { otimizado desenhado. }\end{array}$ & $\begin{array}{l}\text { - Treinamento sobre o novo } \\
\text { procedimento. }\end{array}$ \\
\hline & $\begin{array}{l}\text { - Medir os indicadores após a } \\
\text { implementação da modelagem } \\
\text { de processo e comparar com os } \\
\text { valores anteriores. }\end{array}$ & - KPI (Key Process Indicator) \\
\hline
\end{tabular}

Fonte: Elaboração própria, com base em (Choguill, 2005) 


\section{DESENVOLVIMENTO}

\subsection{Construção de Indicadores}

A execução da modelagem do processo de negócio foi estruturada em um evento KAIZEN, onde a filosofia do pensamento enxuto incentiva que os funcionários busquem continuamente melhores maneiras de se fazer as coisas e consequentemente melhorar a qualidade, eficiência e velocidade (Bruun \& Mefford, 2004).

Estes eventos são fomentados e apoiados pela gerência da empresa e a implementação de mudanças de processos necessita de forte apoio gerencial para ser efetivamente praticada em formato de grupo de foco (Rohleder \& Silver, 1997).

O grupo de foco teve origem na sociologia, no entanto, é amplamente utilizado na área de marketing e com crescente popularidade em outros campos de ação (Hopkins \& Irvine, 2012; Sharts-Hopko, 2001). Consiste em uma entrevista realizada por um moderador bem treinado, de uma forma não estruturada e natural, com um pequeno grupo de respondentes, visando descobrir possíveis ideias ou soluções para um problema por meio da discussão do tema.

A equipe do projeto de modelagem de processos foi formada por representantes das áreas envolvidas na logística do calcário, seguindo a sugestão de (Rohleder \& Silver, 1997), que recomendam que os membros da equipe devam estar envolvidos no processo em questão. As áreas envolvidas no processo são:

Tabela 3: Áreas Envolvidas e Responsabilidades

\begin{tabular}{ccc}
\hline Áreas Envolvidas & Envolvimento no Processo \\
\hline Planejamento integrado & - $\begin{array}{r}\text { Responsável pela confecção do programa de produção, de acordo } \\
\text { com informações de planejamento de vendas, extensão da } \\
\text { campanha, estoque em pátio, restrições de manutenção, maré, etc. }\end{array}$ \\
\hline Operadores logísticos & - $\begin{array}{r}\text { No caso em estudo, uma empresa terceirizada é responsável pela } \\
\text { programação dos insumos junto aos fornecedores e pelo } \\
\text { recebimento e descarregamento das matérias-primas. }\end{array}$ \\
\hline Suprimentos & $\begin{array}{r}\text { Responsável pelo contato direto com o fornecedor, para resolução } \\
\text { de questões comerciais ou de problemas de qualquer ordem. }\end{array}$ \\
\hline
\end{tabular}

Fonte: Elaboração própria.

Na primeira reunião, foi realizado um brainstorming pela equipe, que levantou vários possíveis indicadores do processo logístico do calcário. Após discussão e consenso entre a equipe, ficou estabelecido o seguinte indicador:

- Número de horas paradas dos moinhos de calcário por falta de calcário bruto no silo (P1). O intuito deste indicador é verificar se os moinhos de calcário deixaram de moer o insumo pelo fato do silo estar vazio.

Definido o indicador, o próximo passo foi coletar os dados para verificação dos valores históricos do KPI determinado (Looy, Backer, \& Poels, 2014). O indicador pôde ser calculado a partir dos dados coletados no sistema supervisório da empresa, que descreve especificamente as 
paradas dos moinhos devido à falta de calcário bruto no silo durante 15 meses. A empresa possui dois moinhos de calcário e definiu-se que P1 é a soma das paradas dos moinhos por falta de calcário bruto. A Figura 1 ilustra o indicador P1 para o período estudado.

Figura 1: Horas paradas por falta de calcário bruto.

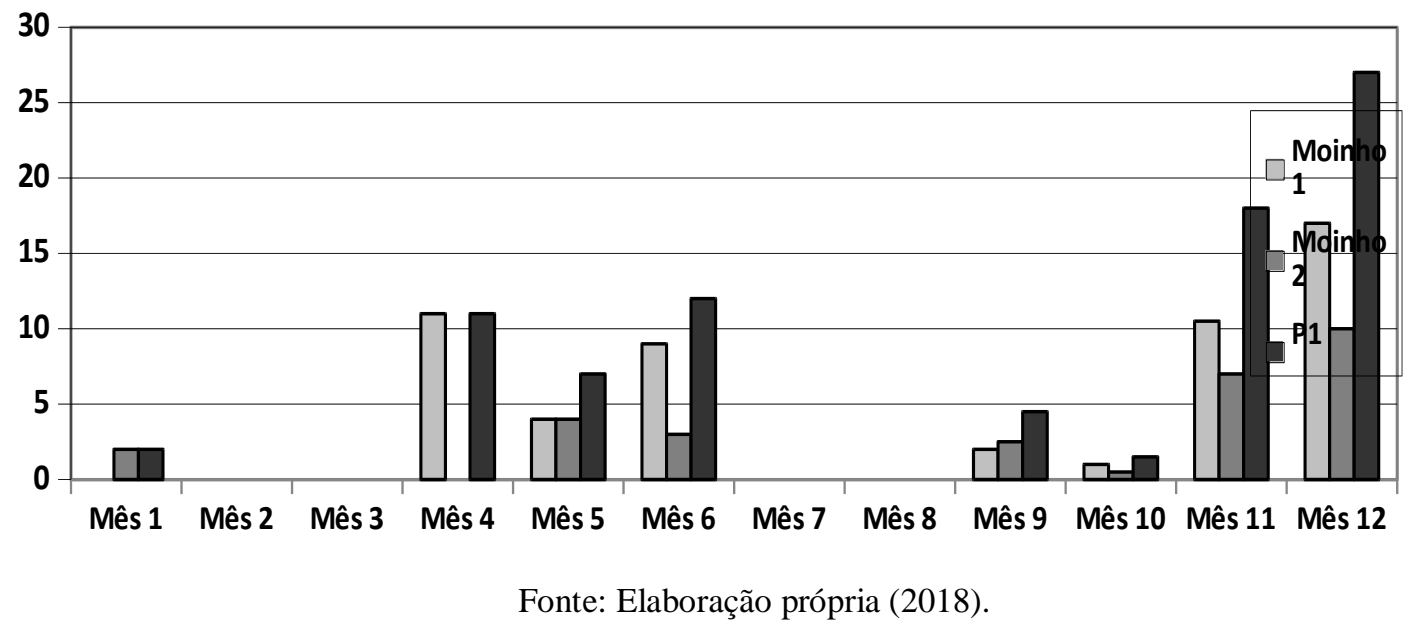

Uma informação importante extraída do gráfico é a média aritmética mensal de P1 no período, que equivale a 6,93 horas. Além disso, observa-se que o perfil do indicador é ascendente.

\subsection{Modelagem do estado atual (As-Is)}

Nesta pesquisa, foi utilizada a notação BPMN, que é considerada a linguagem padrão para modelagem de processos de negócios (Recker, 2010). Um dos motivos para a escolha do BPMN se deve ao fato de ser um método gráfico, já que métodos gráficos revelam facilmente os padrões e gargalos de um processo de negócio (Ko et al., 2009).

O software escolhido para a representação do modelo foi o Visio, da Microsoft, por ser a ferramenta mais popular, conforme (Recker, 2010; Rosemann, 2006). Esses autores acrescentam ainda que esta ferramenta é apropriada para modelar processos pequenos, com propósitos específicos.

A próxima etapa realizada pela equipe de modelagem de processos foi desenhar o processo atual, o As-Is. Nessa reunião, os membros da equipe descreveram o processo atual, utilizando a notação de modelagem BPMN. A Figura 2 ilustra o processo modelado. 
Figura 2: Modelo do processo “Programar e receber calcário” no estado atual (As Is).

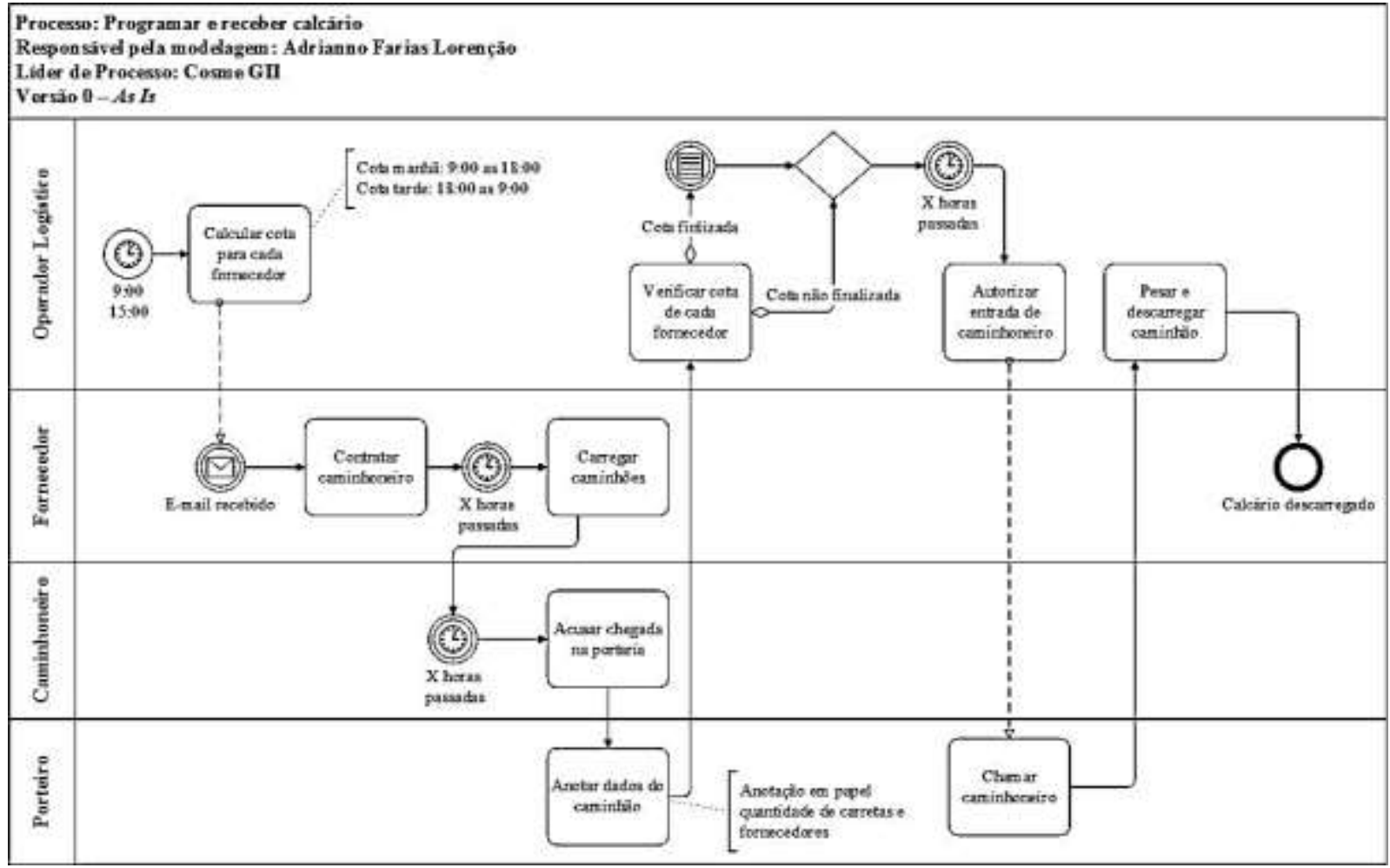

Fonte: Elaboração própria (2018).

\subsection{Modelagem do estado futuro}

Após a consolidação do modelo As-Is entre todos os membros da equipe, nova reunião foi agendada para a discussão e construção do modelo To-Be. Nessa atividade, várias soluções simples para problemas antigos foram levantadas.

Para a elaboração do novo processo, foram discutidas todas as falhas e problemas do modelo atual. Algumas diretivas foram estabelecidas para dar foco à modelagem, tais como: criação de ferramentas para retirar o processo decisório dos operadores; criação de controles, de modo que as variáveis do processo sejam facilmente acessadas e compartilhadas; redução das pessoas envolvidas, para minimizar problemas de comunicação; padronização.

Dentre as principais alterações para a concretização do modelo To-Be, destacam-se:

- Elaborar planilha padrão para cálculo da cota de cada fornecedor, com base nas informações necessárias (estoque, campanhas, etc.);

- Criar e-mail padrão para divulgação das cotas;

- Criar planilha compartilhada entre Operador Logístico, Portaria e Suprimentos;

- Criar planilha para controle dos caminhões recebidos dos fornecedores;

- Implementar sistema remoto de chamada de caminhoneiros para gestão da fila. 
O modelo To-Be construído pela equipe está representado nas Figuras 2 e 3. Optou-se por dividir o processo em dois: programar calcário e receber calcário. A equipe percebeu que ao tratar como processos distintos, ter-se-ia maior clareza e melhor interpretação do modelo por parte dos operadores.

Finalizado o desenho do modelo, todos os envolvidos foram treinados no novo procedimento. Nesse ponto, observou-se que as pessoas com menor nível de instrução, que trabalham na portaria e na balança, tiveram dificuldade para assimilar todas as informações contidas no modelo. Além disso, a execução do novo processo desenhado sofreu um pequeno atraso, pois uma das soluções levantadas (implementação de sistema remoto de chamadas) dependia da compra e instalação de equipamentos.

\section{RESULTADOS}

Para verificar os possíveis ganhos obtidos com a implementação da modelagem de processos de negócios, os dados para construção do indicador P1 foram novamente coletados no sistema supervisório, buscando informações sobre as paradas dos moinhos de calcário.

A Figura 1 ilustra o comportamento do indicador: número de horas paradas dos moinhos de calcário por falta de calcário bruto no silo. Para analisar os dados explicitados nesse gráfico, deve ser considerada a evolução do projeto de modelagem:

- Primeira reunião, para construção dos indicadores: mês 9.

- Segunda reunião, para modelagem As-Is: mês 10.

- Terceira reunião: modelagem To-Be: mês 10

- Treinamento dos envolvidos no novo modelo: mês 11

- Kick-off do novo modelo do processo: mês 12 
Figura 3: Modelo do processo "Programar calcário" no estado futuro (To-Be)

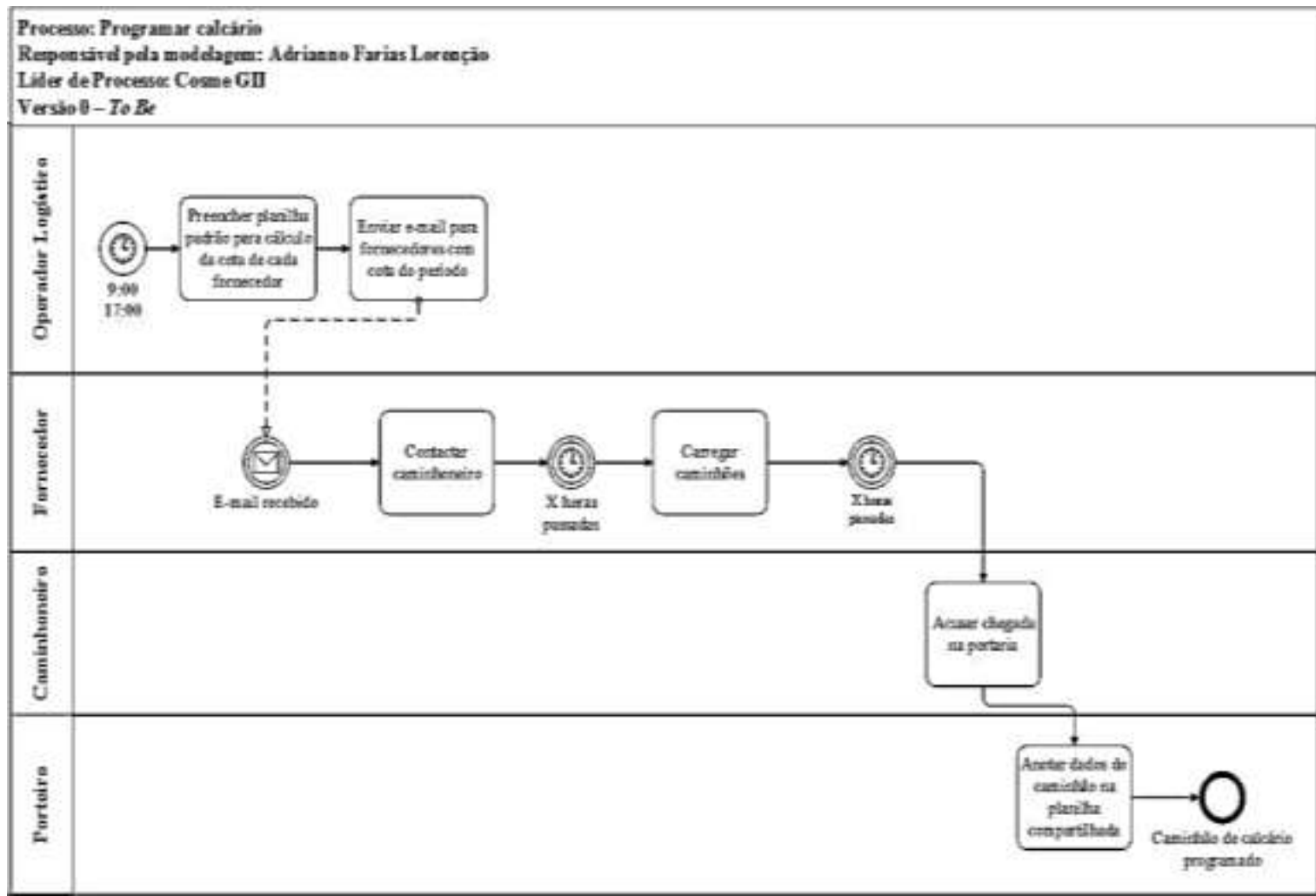

Fonte: Elaboração própria (2018). 
Figura 3: Modelo do processo “Receber calcário” no estado futuro (To-Be).

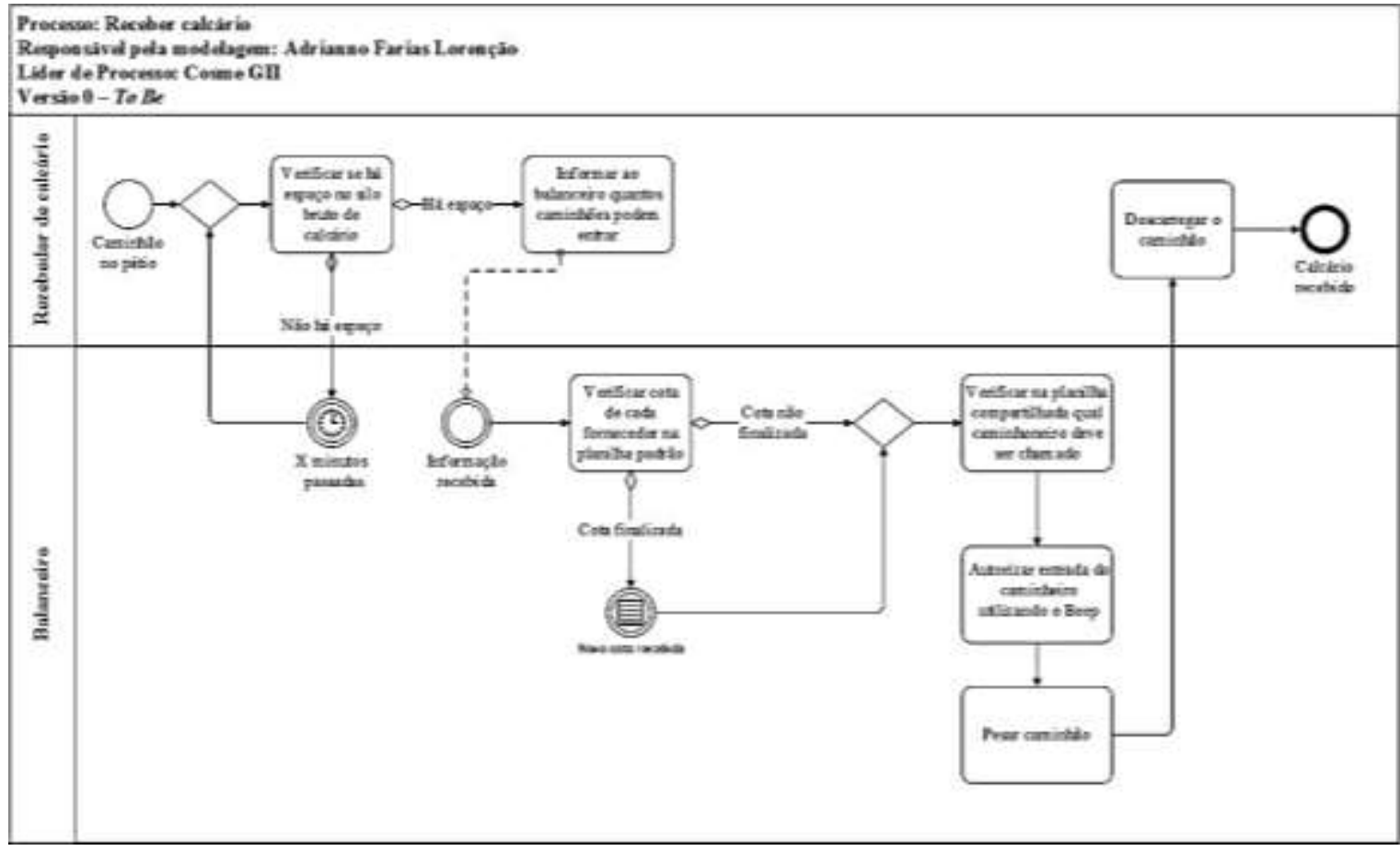

Fonte: Elaboração própria (2018).

Pela análise dos dados explicitados, pode-se inferir que o novo modelo de processo provocou uma melhora no indicador estipulado pela equipe. A média aritmética do KPI na baseline (Set/09 a Ago/10) foi de 6,93h. Nos meses de dezembro e janeiro, quando o processo já havia sido implementado, a média aritmética foi de 1,15h. Ou seja, reduziu-se em seis vezes o número médio de horas paradas por falta de material nos moinhos de calcário. Ademais, a média aritmética do indicador dos quatro meses anteriores à implantação foi de 35,3 horas de paradas por mês. Este dado mostra a queda brusca do indicador no Gráfico 2.

Hachicha et al. (2016), destacam um método de avaliação que usa vestígios em processos combinados com um método de avaliação de alto nível utilizando indicadores-chave de desempenho, através de um estudo de caso industrial, afirmou que a abordagem é capaz de identificar eventos que interrompem o bom funcionamento dos processos no tempo de execução.

Apesar do KPI evidenciar uma melhora no desempenho do processo, dois pontos de atenção devem ser destacados. Primeiramente, verificou-se que o indicador não alcançou a meta previamente estabelecida, que era eliminar as paradas devido à falta do insumo. Outra questão foi o curto período de medição pós-implementação. Foram levantadas dúvidas se dois meses de controle seriam suficientes para afirmar que o novo processo responderá a contento a todas as perturbações. Entretanto, na literatura, não é evidenciado o tempo necessário para que o processo saia do estado transiente, já que a magnitude deste período é função de inúmeras variáveis do processo que está sendo estudado. Essas questões, todavia, não desabonam a confirmação de que a modelagem de processos promoveu melhorias.

Foram enfrentadas algumas dificuldades no treinamento da equipe operacional no novo procedimento. A linguagem BPMN, apesar de se mostrar bastante flexível, não foi facilmente 
assimilada. O principal motivo foi o desconhecimento do significado dos diagramas que representavam os eventos. Caso o escopo do BPM seja mais amplo e englobe muitos processos da empresa, faz-se necessário o treinamento básico de todos os empregados na linguagem BPMN. Apesar de amplamente divulgado, o método ainda sofre resistência no meio empresarial por gerar dúvidas se agrega valor suficiente, dado o esforço para implementá-lo.

Destaca-se, finalmente, que o escopo do BPM descrito neste artigo foi pontual, ou seja, apenas um processo foi avaliado. Sugere-se um estudo desta natureza numa implementação mais abrangente de BPM, em que outras dificuldades poderão ser encontradas e os resultados podem diferir do que foi exposto neste artigo. Além disso, conforme afirmam (Vom Brocke et al., 2014), existem vários métodos para modelagem de processos de negócios e nenhuma abordagem deve ser a priori considerada a mais adequada para a aplicação. A melhor opção deve ser o uso integrado de várias técnicas.

\section{CONCLUSÃO}

Os resultados encontrados comprovaram-se positivos para o processo estudado, ao avaliar os ganhos obtidos na implementação de modelos de processos de negócio. Portanto, a abordagem ontológica utilizada pode direcionar a avaliação de processos de negócios colaborativos de forma eficaz.

Nos meses anteriores à implementação do novo modelo de processo, a média de horas paradas do moinho de calcário por falta de calcário bruto (indicador estabelecido para o processo) foi de $35,3 \mathrm{~h}$. Este valor caiu para 1,15h nos meses posteriores ao trabalho, evidenciando uma melhora na logística do insumo para pelotização do minério de ferro. É importante ressaltar também que, apesar do novo processo ainda não ter sido implementado, verificou-se uma abrupta mudança no indicador de 49,52h de parada no Mês 14 para 5,55h de parada no Mês 15. Atribui-se essa melhora à percepção dos envolvidos de que o processo estava sendo estudado, controlado, gerido, o que gerou uma preocupação para obter melhores resultados. Depreende-se daí que, para um processo proporcionar um bom desempenho, é imprescindível que haja um envolvimento de todos os colaboradores.

\section{REFERÊNCIAS BIBLIOGRÁFICAS}

Aguilar-Savén, R. S. (2004). Business process modelling: Review and framework. International Journal of Production Economics, 90(2), 129-149. https://doi.org/10.1016/S09255273(03)00102-6

Bruun, P., \& Mefford, R. N. (2004). Lean production and the Internet. International Journal of Production Economics, 89(3), 247-260. https://doi.org/10.1016/j.ijpe.2003.10.007

Choguill, C. L. (2005). The research design matrix: A tool for development planning research studies. Habitat International, 29(4), 615-626. https://doi.org/10.1016/j.habitatint.2005.06.001 
Conforti, R., De Leoni, M., La Rosa, M., Van Der Aalst, W. M. P., \& Ter Hofstede, A. H. M. (2015). A recommendation system for predicting risks across multiple business process instances. Decision Support Systems, 69, 1-19. https://doi.org/10.1016/j.dss.2014.10.006

Conforti, R., Dumas, M., García-Bañuelos, L., \& La Rosa, M. (2016). BPMN Miner: Automated discovery of BPMN process models with hierarchical structure. Information Systems, 56, 284303. https://doi.org/10.1016/j.is.2015.07.004

Corradini, F., Ferrari, A., Fornari, F., Gnesi, S., Polini, A., Re, B., \& Spagnolo, G. O. (2018). A Guidelines framework for understandable BPMN models. Data and Knowledge Engineering, 113(November 2016), 129-154. https://doi.org/10.1016/j.datak.2017.11.003

Dijkman, R., Lammers, S. V., \& de Jong, A. (2016). Properties that influence business process management maturity and its effect on organizational performance. Information Systems Frontiers, 18(4), 717-734. https://doi.org/10.1007/s10796-015-9554-5

Faé Gomes, G. M., Vilela, A. C. F., Zen, L. D., \& Osório, E. (2013). Aspects for a cleaner production approach for coal and biomass use as a decentralized energy source in southern Brazil. Journal of Cleaner Production, 47, 85-95. https://doi.org/10.1016/j.jclepro.2012.09.037

Hammer, B. M. (1993). Reengineering the corporation: a manifesto for business revolution [Book Review]. IEEE Engineering Management Review, 31(4). https://doi.org/10.1109/EMR.2003.1267014

Hopkins, J., \& Irvine, F. (2012). Qualitative insights into the role and practice of Epilepsy Specialist Nurses in England: A focus group study. Journal of Advanced Nursing, 68(11), 2443-2453. https://doi.org/10.1111/j.1365-2648.2012.05941.x

Ko, R. K. L., Lee, S. S. G., \& Lee, E. W. (2009). Business process management (BPM) standards: A survey. Business Process Management Journal (Vol. 15). https://doi.org/10.1108/14637150910987937

Launonen, M., \& Kess, P. (2002). Team roles in business process re-engineering. International Journal of Production Economics, 77(3), 205-218. https://doi.org/10.1016/S09255273(00)00158-4

Looy, A. Van, Backer, M. De, \& Poels, G. (2014). A conceptual framework and classification of capability areas for business process maturity. Enterprise Information Systems, 8(2), 188-224. https://doi.org/10.1080/17517575.2012.688222

Luo, W., \& Tung, Y. A. (1999). A framework for selecting business process modeling methods. Industrial Management and Data Systems, 99(7), 312-319. https://doi.org/10.1108/02635579910262535

Mitsyuk, A. A., Shugurov, I. S., Kalenkova, A. A., \& van der Aalst, W. M. P. (2017). Generating event logs for high-level process models. Simulation Modelling Practice and Theory, 74, 1-16. https://doi.org/10.1016/j.simpat.2017.01.003 
Recker, J. (2010). Opportunities and constraints: The current struggle with BPMN. Business Process Management Journal, 16(1), 181-201. https://doi.org/10.1108/14637151011018001

Rohleder, T. R., \& Silver, E. A. (1997). OPERATIONS Tutorial A tutorial on business process improvement. Desiciones Estrategicas, 6963(96).

Rosemann, M. (2006). Potential pitfalls of process modeling: Part B. Business Process Management Journal, 12(3), 377-384. https://doi.org/10.1108/14637150610668024

Schmiedel, T., Müller, O., \& vom Brocke, J. (2018). Topic Modeling as a Strategy of Inquiry in Organizational Research: A Tutorial With an Application Example on Organizational Culture. Organizational Research Methods, (April). https://doi.org/10.1177/1094428118773858

Sharts-Hopko, N. C. (2001). Focus group methodology: when and why? The Journal of the Association of Nurses in AIDS Care : JANAC, 12(4), 89-91. https://doi.org/10.1016/S10553290(06)60220-3

Solís-Martínez, J., Espada, J. P., Pelayo G-Bustelo, B. C., \& Lovelle, J. M. C. (2014). BPMN MUSIM: Approach to improve the domain expert's efficiency in business processes modeling for the generation of specific software applications. Expert Systems with Applications, 41(4 PART 2), 1864-1874. https://doi.org/10.1016/j.eswa.2013.08.083

Souza, M. C., Saueressig, G. G., Gustavo Junior, J. U., Luchese, J., Bauer, J. M., \& Sellitto, M. A. (2017). Identificação De Perdas Em Processo De E-Commerce Segundo O Referencial Do Sistema Toyota De Produção. Holos, 8, 192. https://doi.org/10.15628/holos.2016.3191

Van Der Aalst, W. M. P., La Rosa, M., \& Santoro, F. M. (2016). Business process management: Don't forget to improve the process! Business and Information Systems Engineering, 58(1), 1-6. https://doi.org/10.1007/s12599-015-0409-x

Vergidis, K., Turner, C. J., \& Tiwari, A. (2008). Business process perspectives: Theoretical developments vs. real-world practice. International Journal of Production Economics, 114(1), 91-104. https://doi.org/10.1016/j.ijpe.2007.12.009

Vom Brocke, J., Schmiedel, T., Recker, J., Trkman, P., Mertens, W., \& Viaene, S. (2014). Ten principles of good business process management. Business Process Management Journal, 20(4), 530-548. https://doi.org/10.1108/BPMJ-06-2013-0074

Yen, V. C. (2009). An integrated model for business process measurement. Business Process Management Journal, 15(6), 865-875. https://doi.org/10.1108/14637150911003757

Yousfi, A., Saidi, R., \& Dey, A. K. (2016). Variability patterns for business processes in BPMN. Information Systems and E-Business Management, 14(3), 443-467. https://doi.org/10.1007/s10257-015-0290-7 